\title{
Lidil
}

Revue de linguistique et de didactique des langues

Acquisition et enseignement de la morphographie

\section{Morphographie et production d'écrits au cycle 3 des écoles}

Jean-Christophe Pellat et Gérard Teste

\section{OpenEdition}

Journals

Édition électronique

URL : http://journals.openedition.org/lidil/783

DOI : $10.4000 /$ lidil.783

ISSN : $1960-6052$

Éditeur

UGA Éditions/Université Grenoble Alpes

Édition imprimée

Date de publication : 1 décembre 2004

Pagination : 87-100

ISBN : 2-914176-11-2

ISSN : $1146-6480$

\section{Référence électronique}

Jean-Christophe Pellat et Gérard Teste, « Morphographie et production d'écrits au cycle 3 des écoles », Lidil [En ligne], 30 | 2004, mis en ligne le 29 janvier 2008, consulté le 03 mai 2019. URL : http://

journals.openedition.org/lidil/783 ; DOI : 10.4000/lidil.783

(c) Lidil 


\title{
MORPHOGRAPHIE ET PRODUCTION D'ÉCRITS
} AU CYCLE 3 DES ÉCOLES

\author{
Jean-Christophe PELLAT* et Gérard TESTE **
}

La morphographie constitue sans doute une des difficultés majeures dans l'apprentissage de l'orthographe française, et peut être encore une cause non négligeable d'erreurs chez le scripteur adulte expert. Pour guider les élèves vers la maitrise de l'orthographe, la démarche classique (observation rapide, induction immédiate d'une règle abstraite suivie d'exercices d'application) ne suffit pas: il est nécessaire de donner aux élèves l'occasion d'écrire «pour de bon » si l'on veut les aider à acquérir des automatismes graphiques, grâce à un va-etvient entre la pratique de l'écriture et le retour réflexif sur cette pratique. Telle était l'orientation fondamentale de l'expérience que nous avons conduite, pendant plusieurs années, dans le cadre d'une recherche-action, menée avec le soutien de l'IUFM d'Alsace, portant sur l'acquisition des chaines morphographiques d'accord au cycle 3 des écoles. Une équipe de huit enseignants a élaboré et réalisé dans six classes de cycle 3 des moments de découverte et de structuration visant à articuler l'apprentissage de la morphographie avec les pratiques d'écriture, qui assurent la mise en œuvre effective des règles orthographiques. Les principes méthodologiques adoptés ont été les suivants: pas de démarche de type expérimental visant une administration de la preuve irré-

* Université Marc Bloch-Strasbourg 2.

** IUFM d'Alsace. 
futable, mais plutôt une démarche en trois temps incluant tout d'abord un volant de conception et d'élaboration de séquences didactiques, ensuite un volant de passation de séquences en situation réelle de classe, enfin un volant de retour réflexif et d'élaboration de points d'appui invariants pour la pratique, inspiré de sources théoriques empruntées ou élaborées et de savoir empirique. Cette recherche a abouti à une publication au CRDP d'Alsace, Orthographe et écriture: pratique des accords (Pellat et Teste, 2001). Nous allons présenter les principes didactiques et pédagogiques qui ont guidé cette recherche, puis nous évoquerons les activités mises en œuvre pour faciliter l'apprentissage de la morphologie en associant pratiques d'écriture et réflexion métagraphique.

\section{Orientations didactiques et pédagogiques}

\section{Constats}

Le point de départ de la recherche engagée réside dans ce constat souvent fait par les enseignants et repris à son compte par l'équipe de recherche: au cours des tâches d'écriture qui leur sont demandées en classe ou en travail à la maison, les élèves, lorsqu'ils doivent faire appel à un savoir-faire précis, par exemple savoir marquer correctement les accords propres à lune chaine morphographique précise, ont beaucoup de difficultés non seulement à en apercevoir les lieux d'application dans leur écrit en cours, mais encore à convoquer le souvenir de la règle ou de la régularité de fonctionnement qui a été mise en évidence lors des leçons de découverte et de structuration effectuées en classe autour de ce savoir-faire partiel.

Si l'on ajoute à ce constat le fait que, comme l'attestent de multiples témoignages d'enseignants, les élèves réussissent plutôt bien que mal les exercices d'application qui clôturent la plupart du temps ces leçons, cette réussite très localisée au moment des exercices masque en fait leur difficulté à progresser dans l'acquisition de l'orthographe en actes: cet écart entre une mobilisation satisfaisante des acquis lors des moments institutionnalisés d'observation réfléchie de la langue et une appropriation problématique des savoir-faire 
visés, observée dans les écrits empiriques complexes demandés aux élèves, est souvent relevé par l'opinion commune qui se demande parfois pourquoi, en dépit de nombreuses heures passées en classe à travailler l'orthographe et la grammaire, les savoir-faire rédactionnels des élèves sont si décevants. Quant aux élèves, ceux du moins qui, à l'école, ne sont pas suffisamment confrontés à des productions d'écrits quotidiens en diverses situations, ils peuvent fort bien vivre toute leur scolarité sans avoir été amenés à prendre conscience de cet écart. Ils font alors des «activités», comme on dit, de grammaire, d'orthographe, acquièrent à la longue des tours de main pour réussir dans ces situations très contextualisées et n'en retrouvent pas l'opérativité dans d'autres situations où ces tours de main auraient précisément à s'exercer. Ils en restent alors au niveau du «faire pour faire », encouragés parfois en cela par une pratique obsédante de l'évaluation qui parcellise et atomise les savoirs, au nom d'une certaine rigueur dans la détermination des objectifs, alors qu'il conviendrait tout au contraire d'évaluer leur appropriation de manière intégrative.

Si nous voulons à présent analyser ce qui peut expliquer ce constat fréquemment effectué, nous pouvons déceler quatre zones d'ombre dans le trajet d'une leçon de structuration telle qu'elle est habituellement pratiquée.

Pour en rappeler sommairement le trajet, une brève phase d'observation initiale, collective la plupart du temps, est effectuée sur un fait de langue à l'aide de quelques exemples portés au tableau ou repris du manuel. Cette phase initiale est suivie de l'énonciation orale/écrite de la régularité soumise à observation et codifiée ensuite sous la forme d'une règle ou toute autre forme de conceptualisation, tableau classant par exemple: cette seconde phase codifie par induction les régularités observées. Les exercices cumulés suivis, le cas échéant, d'exercices de remédiation, constituent la troisième phase d'application par déduction. Est visé ensuite le détachement du savoir-faire et du savoir dans les situations d'orthographe en actes, ultérieures à la leçon.

Des quatre zones d'ombre inhérentes à la logique même de ce trajet, la première se situe dès la phase d'observation initiale, problématique en soi : on n'observe bien que ce sur quoi l'on s'est posé des questions au préalable; or en phase ini- 
tiale, mis à part un tout petit nombre d'élèves co-dirigeants de la classe qui ont déjà compris l'enjeu de l'observation demandée, il est très difficile à la plupart d'observer un fait de langue dont il s'agit précisément d'établir la régularité à partir d'un nombre très restreint d'exemples.

Une seconde zone d'ombre se situe à la jonction des deux premières phases, entre l'observation et l'induction d'une régularité codifiable; à ce moment, le saut inductif demandé aux élèves est trop grand, trop hâtif, trop tôt venu, pour ne pas générer une grande confusion cognitive.

Une troisième zone d'ombre est paradoxalement liée à la réussite remarquée aux exercices d'application: ils sont à la fois nombreux et variés et, même s'ils sont relativement bien réussis en raison de leur caractère très apprêté et de leur grande proximité à la forme du savoir mis sous forme de règle dans la phase précédente, ils ne garantissent en rien une appréhension suffisante de l'objectif visé par la leçon; quant aux exercices de remédiation ultérieurs pour ceux des élèves qui n'auraient pas satisfait aux travaux d'application, les témoignages empiriques des maitres concordent pour dire que ce n'est pas «en donnant toujours plus du même», selon une dynamique identique à celle de l'application, que la compréhension d'un fonctionnement grammatical régulier de la langue peut s'effectuer.

La quatrième zone d'ombre concerne la capacité de détachement du savoir acquis et sa reconnaissance iors des moments d'orthographe en actes: les savoir-faire à mobiliser ne sont que partiellement reconnus en raison du caractère très contextualisé des activités de découverte et de structuration, en raison également du fait qu'ils n'ont pas été suffisamment mis en mots par les élèves, à leur manière et selon leur degré de compréhension.

\section{Démarche de découverte el de structuration}

Dans la conception et l'élaboration des séquences de structuration de la langue, notre équipe de recherche a travaillé sur ces zones d'ombre et ce, dans les directions suivantes: en phase initiale, les modifications suivantes sont introduites: 
l'observation collective est remplacée par une observation active sous la forme de demande de tâche à accomplir, du type «mini-atelier d'écriture». Ainsi, pour travailler l'accord du verbe au passé composé avec le sujet selon que l'auxiliaire est être ou avoir, on a demandé aux élèves de rédiger de brefs récits litaniques incluant un passé composé à chaque proposition sur les quatre thèmes suivants (au choix):

Les pompiers ont fait des exercices

Un enfant s'est perdu à New York

La voiture a grillé un feu rouge

Un homme masqué est entré dans la banque.

De ce fait, un travail individuel ou par dyades est privilégié par rapport à un travail de réflexion à haute voix en collectif anonyme ; l'étendue du fait de langue observé est modifiée ainsi que le temps consacré à cette première phase, ce qui suppose l'instauration d'habitudes de travail en société coutumière de classe. En raison de ces modifications, la nature même de l'observation visée est transformée.

La source du «texte du savoir» est constituée du produit des «mini-ateliers d'écriture» : voici un exemple de texte produit à partir du premier inducteur ci-dessus Les pompiers ont fait des exercices:

\section{Ils ont éteint un feu}

Ils sont montés sur un immeuble

Ils sont passés par une fenêtre

Ils ont dégagé des mannequins

Ils se sont entrainés à s'habiller.

Le savoir induit provient de ces manipulations préalables, s'appuyant sur les opérations linguistiques d'adjonction, d'effacement, de déplacement, de substitution. Ce savoir est élaboré sous la forme d'une confrontation collective obtenue à partir des découvertes et médiatisée nécessairement par le maitre. Il ne se présente pas sous la forme d'une règle puissante et abstraite mais il est plutôt la trace de ce que les élèves ont été en mesure de "dire sur le fait de langue observé»; il garde par conséquent la trace du processus de découverte et peut avoir des formes variées: tableaux classant à fonction analogique par exemple, exemples illustratifs jugés à la fois éloquents et significatifs. 
La troisième phase d'exercices d'application n'est pas régie par une logique de type cumulatif d'une part, et d'autre part, elle est rapportée aussi étroitement que possible au savoir élaboré en phase deux ci-dessus. Ces exercices d'application sont évalués systématiquement en avec Jean-Pierre Jaffré (Ducard et al., $1995: 10$ ) que classe même et fournissent ainsi l'occasion de «dire des choses» sur la langue et son système.

En ce qui concerne le détachement des savoirs acquis en termes de savoir-faire au service de l'écriture, si l'on veut bien admettre "production écrite et savoirs métagraphiques constituent les deux moteurs de l'acquisition de l'orthographe», les outils artisanaux de classe du type cahiers de «gammes d'écriture » ou «carnets de langue » sont utilisés de manière systématique comme référents dans les situations d' «écriture pour de bon». Dans le même ordre d'idées, la pratique de la correction différée, par le maitre, est la façon de faire au quotidien: en situation d'écriture, les élèves, sur consigne précise donnée, sont invités à rédiger un premier jet prenant en compte le respect de la consigne et les préoccupations de continuité textuelle concernant notamment les phénomènes de cohérence, de cohésion et de connexité. D'un point de vue didactique, nous trouvons utile le recours à ces trois notions: il permet en effet d'aiguiser le regard en retour des élèves sur les productions de leurs pairs et sur les leurs propres: ainsi, un écrit premier jet peut être parfaitement cohérent et en même temps peu satisfaisant du point de vue des marqueurs linguistiques de cohésion et de connexité qui y sont manifestes; inversement, un texte bien formé du point de vue cohésif et «rempli»de connecteurs selon la vulgate des nouveaux manuels d'observation réfléchie de la langue peut être difficilement recevable en lecture en raison des phénomènes d'incohérence qui s'y manifestent.

Les premiers jets ne sont pas immédiatement repris par le maitre pour annotation et évaluation éventuelles; ils sont retravaillés quelque temps après par leurs auteurs sur l'invitation de l'enseignant qui leur indique alors que des savoirfaire, orthographiques entre autres, ont été laissés de côté dans le cours de la rédaction initiale. C'est l'occasion pour les 
élèves de revenir sur leurs écrits à l'aide des usuels et de leurs outils du type «cahier de gammes d'écriture».

Cette articulation entre des situations scolaires de productions écrites aménagées à partir des pratiques sociales de référence et des dispositifs didactiques permettant de découvrir et de structurer les savoirs et les savoir-faire partiels pour mener à bien les tâches complexes de production nous parait être le point nodal de l'apprentissage en matière de maitrise de la langue. Par delà les modifications, relatées ci-dessus, affectant les trajets de séance en matière d'observation réfléchie de la langue, il nous apparait que trois principes, entendus non pas au sens aristotélicien mais au sens kantien du terme, c'està-dire des lignes de conduite auxquelles on peut se référer pour avancer et justifier des propositions didactiques, peuvent être légitimement respectés en la matière, les principes d'homologie, de détachement et d'extensivité.

Le principe d'homologie entre les situations d'écriture pour de bon et les situations de structuration de savoir-faire partiels conduit à élaborer une nouvelle classe d'exercices du type «productions écrites-problèmes»: comme on l'a montré à l'aide de l'exemple ci-dessus, les très brefs écrits produits dans ce cadre au tout début des séances de structuration ressemblent à des écrits véritables tout en spectacularisant l'objectif-obstacle visé. Le principe de détachement aménage quant à lui le passage de l'observation des faits de langue à la codification de leur régularité sous une forme adaptée à la manière dont les élèves parlent de la langue quand la latitude leur en est laissée; cette forme adaptée consignée dans un outil du type carnet de "gammes d'écriture» constitue le texte du savoir provisoire dont l'usage sera systématiquement sollicité en situation de production ultérieure. Le principe d'extensivité se traduit en termes de programmation à l'intérieur du cycle et permet, lors de la rencontre fortuite ou de la découverte de formes jamais observées jusqu'alors de chaines morphographiques d'accords, de rapporter l'inconnu au connu par extension de la régularité à la nouvelle forme considérée ${ }^{1}$. 


\section{L'apprentissage des accords}

\section{Des difficultés de la morphographie}

La complexité de l'orthographe française est due, pour une grande part, à la dimension morphographique et à ses interférences avec les dimensions phonographique et logogrammique, ce qui donne lieu à de nombreuses difficultés d'apprentissage. En outre, dans la progression de l'acquisition de l'orthographe, les erreurs les plus tenaces concernent la morphographie, y compris chez les scripteurs adultes, en raison des particularités de son fonctionnement. Celle-ci s'articule en partie avec la phonographie. En premier lieu, certains graphèmes, les morphonogrammes, ont une double valeur, phonographique et morphographique: ainsi, dans il chantait, le digramme $a i$ correspond au phonème $[\varepsilon]$ et constitue la marque caractéristique de l'imparfait de l'indicatif. Il semble que ces morphogrammes permettent les premières découvertes morphographiques dans l'apprentissage de l'orthographe: dès le CP, les enfants perçoivent que l'emploi des phonogrammes peut être déterminé par des contraintes morphologiques (Ducard et al., 1995 : 149; Jaffré, 1998). En second lieu, le choix de certains morphogrammes peut s'appuyer sur la dimension phonographique: ainsi, dans chevaux, le $-x$ du pluriel, non prononcé sauf en cas de liaison, est associé au phonogramme au, et constitue avec lüi une séquence graphique stable, qui s'oppose globalement à la finale -al du singulier. Et puis, les morphogrammes lexicaux des mots de base, non prononcés, sont motivés par leur prononciation dans les dérivés (comme le $d$ dans tard/retarder); l'exploitation des familles de mots facilite l'apprentissage de l'orthographe lexicale. Mais beaucoup de morphogrammes grammaticaux sont des lettres muettes, sans relation directe ou indirecte avec la phonographie. On entre alors dans la dimension purement visuographique de l'orthographe française. L'apprentissage de la morphographie rencontre alors deux difficultés. D'un côté, un graphème donné peut prendre plusieurs valeurs grammaticales: ainsi, le graphème -s indique, selon la classe grammaticale, le pluriel des éléments nominaux (déterminants, adjectifs, substantifs: quelques 
pauvres filles) ou la deuxième personne du singulier des verbes ( $t u$ chantes). L'apprentissage des marques du nombre du nom et du verbe est progressif (Fayol et al., 1997): la finale nominale $-s$ (les lettres) est acquise plus tôt que la finale verbale - $n t$ (chantent), et la progression est émaillée de nombreuses erreurs $(* i l s$ chantes), qui peuvent même se rencontrer dans des écrits d'adultes, en particulier quand une même forme peut être, selon sa distribution, un nom ou un verbe (*ils timbres les lettres). A cette homographie s'ajoute, d'un autre côté, le phénomène de la redondance graphique des marques grammaticales, principalement du genre et du nombre, alors que l'oral tend à économiser les marques: dans le meilleur des cas, un ou plusieurs indicateurs oraux manifestent le féminin ou le pluriel, le plus souvent le déterminant, comme [le] dans les enfants jouent ; mais, dans certains cas, c'est le contexte large qui apporte l'indication du pluriel, comme dans ils jouent, où l'on doit s'appuyer sur le sens de l'antécédent du pronom $i l$, s'il est anaphorique. Dans tous les cas, l'analyse grammaticale est indispensable, ce qui implique l'acquisition de savoirs morphologiques explicites. Mais, comme nous l'avons vu, ceux-ci constituent une condition nécessaire, mais non pas suffisante à la maitrise de la morphographie.

Parmi les différents aspects de la morphographie, nous avons choisi de travailler sur l'apprentissage des accords grammaticaux, qui représentent une difficulté générale pour les élèves, malgré une base sémantique générale apparemment simple (genre et nombre); la maitrise de ces accords comprend à la fois la sélection de l'affixe final approprié et le marquage de chaque élément appartenant à une même chaine d'accord. Pour cette notion, nous nous référons à une recherche menée naguère en classes de sixième et de cinquième du collège français (11-12 ans) et présentée brièvement par Jean-Pierre Jaffré et Daniel Bessonnat (1993). Nous avons fait nôtres les principes méthodologiques choisis (il était déjà question de l'articulation entre la réflexion grammaticale et l'écriture), en les mettant en œuvre au niveau du cycle 3 des Ecoles. Nous résumons à notre tour les orientations suivies. En se limitant au cadre de la phrase, on désigne sous l'appellation de chaines morphographiques «les suites 
d'éléments qui entretiennent une solidarité morphologique entre eux» (Jaffré et Bessonnat, 1993 : 28).

Dans la phrase Les enfants marchent dans la forêt (fiche 1), on repère deux chaines:

- les enfants marchent: chaine marquée à trois éléments : déterminant + nom constituant le groupe nominal sujet, verbe marcher au présent;

- la forêt : chaine marquée à deux éléments, le déterminant et le nom.

Les chaines morphographiques sont constituées de plusieurs structures syntaxiques: chaines $\mathrm{N}$ (à noyau nominal), composées obligatoirement d'un nom; chaines GN (groupe nominal); chaines GNS + V (groupe nominal sujet + verbe), ou GNS + V être + Attr. (Groupe nominal sujet + verbe être + attribut du sujet). La perception de l'accord est facilitée quand certains éléments (les déterminants et des verbes comme être ou avoir) portent la marque du nombre à l'oral. On postule donc que certaines positions privilégiées, qui peuvent être épargnées par les erreurs, jouent le rôle de pivots dans les chaines d'accords. Partant de ces pivots, Jean-Pierre Jaffré et Daniel Bessonnat (1993 : 28-29) ${ }^{2}$ font intervenir «trois critères contrastifs» pour prévoir «les risques potentiels d'erreurs»:

- le critère de dynamique: le mouvement de l'écriture allant vers la droite en français, les éléments placés à la droite du pivot pourront s'accorder plus «naturellement» dans cette progression. En revanche, un élément placé à gauche du pivot demande une anticipation de l'accord. C'est le cas notamment de l'emploi du prédéterminant tout (tous les enfants sont per$d u s$ ), d'une apposition antéposée au groupe nominal (Égarés, les enfants marchent au hasard), etc.;

- le critère de position: plus la chaine graphique est longue, plus la réalisation de l'accord est difficile: dans Les enfants étaient perdus, l'accord du participe passé perdus,

2. Hormis les déterminants, les variations morphologiques des adjectifs ou des noms marquées à l'oral, comme dans (les chevaux/le cheval), constituent également une aide à la perception de l'accord (Ducard et al. 1995 : 155, encart 42). 
sans marque du pluriel à l'oral et le plus éloigné du pivot les, est plus difficile à réaliser;

- le critère de rupture: la succession des éléments d'une chaine d'accord peut être interrompue par un rupteur, "qui obéit à d'autres lois morphologiques, sans pour autant stopper les effets séquentiels de la chaine» (Jaffré et Bessonnat, 1993 : 28-29). Ce rupteur peut être seulement désactivant, quand il ne porte aucune marque particulière, comme un adverbe (les enfants, très vite, arrivent à la forêt), un pronom relatif simple (les enfants qui courent...), etc. Le rupteur peut être distracteur quand il porte des marques différentes de la chaine d'accord dans laquelle il s'insère; c'est souvent le cas dans les accords «de proximité" (*l'enfant les regardent).

Ainsi, la succession des mots conditionne la réalisation de l'accord, et peut expliquer une bonne partie des erreurs observées, même chez l'adulte expert.

\section{Propositions d'activités}

Les recherches menées dans des classes du cycle 3 des Ecoles ont donné lieu à l'élaboration commune d'activités que nous présentons en dix fiches pratiques:

1.... march (e), (es), (ent)?

2 .... bleu (), bleu (s), bleu (es)

3. Beurre ou confiture?

4. Histoires au passé composé

5. Jeu des mots qui s'enchainent

6. Par la fenêtre I

7. Par la fenêtre II

8. Ecrire un portrait en classe de CE2

9. Ecrire pour inviter à lire

10. La conjugaison par les textes

11. Repères pour agir...

Les fiches 1 à 10 présentent des situations d'apprentissage associant l'écriture et la réflexion orthographique, suivant un dispositif d'ensemble présenté dans la fiche 11 , que nous estimons généralisable à d'autres séquences de travail sur la langue. À titre d'illustration, nous évoquons rapidement les fiches 3 et 6 . 
Fiche 3: Beurre ou confiture?

Les élèves sont invités à lire des devinettes, et à en trouver les solutions à l'aide d'indices orthographiques d'accords. Ils en produisent à leur tour en employant les règles d'accord, celle notamment concernant l'adjectif en position d'attribut :

Devinette $\mathrm{n}^{\circ} 1$

Délicieuse au petit déjeuner, je m'étale sur le pain.

Je suis... le beurre ou la confiture?

Devinette $\mathrm{n}^{\circ} 2$

Bleue le jour

noire la nuit

je suis... la mer ou le ciel?

Nous proposons ci-dessus deux exemples différents de productions. Dans la première devinette, la différence de forme orale constitue une aide pour trouver la réponse, ce qui n'est pas le cas dans la seconde devinette où des formes homophoniques, en français septentrional du moins, sont hétérographiées. Par expérience, les enseignants savent que c'est par les formes hétérophones de l'adjectif qu'il faut commencer la structuration de l'accord de l'adjectif dans la chaine GN.

Fiche 6: Par la fenêtre I

A partir d'un inducteur d'écriture «Par la fenêtre... », les élèves produisent un écrit descriptif: les groupes nominaux sujets indiquent ce qu'on observe par la fenêtre, et sont suivis de groupes du verbe.

On trouvera ci-après un exemple de production premier jet:

La Tour de l'Europe est *présenté en grande hauteur *est en largeur.

L'église que je vois est moderne et entre quelques arbres *est sapins...

L'église St $*$ Paule et Pierre brille au reflet du soleil.

Le ciel *et d'un bleu clair où *quelque nuages blancs s'y promènent.

Cette production et les autres font l'objet d'observations, de classements visant la codification des régularités observées; les GN sujets sont classés en quatre catégories selon la varia- 
tion de genre et de nombre; les exemples des textes sont placés dans la catégorie dont ils relèvent à partir du repérage des pivots $l e, l a, d e s$, un, une, $l$ ': les savoirs métagraphiques sont élaborés à partir des fragments prélevés dans les productions écrites.

\section{Conclusion : pour une diversification des activités d'apprentissage}

L'articulation des activités sur la langue dans l'organisation générale de l'apprentissage du français est actuellement en débat, en particulier au Collège ${ }^{3}$, où l'objectif principal est la production d'écrits, ce qui implique la finalisation des activités de structuration de la langue et ce qui se traduit par l'encouragement au travail en séquences. Dans cette perspective, le dispositif que nous avons présenté illustre une approche intégrée de l'orthographe, mise en relation avec l'activité d'écriture. Cependant, nous n'excluons pas l'approche spécifique, qui reste nécessaire pour construire une progression des apprentissages. C'est pourquoi nous rejoignons la proposition de Catherine Brissaud et Daniel Bessonnat, qui, après avoir examiné les enjeux des modèles «cumulatif ascendant», classique, et «intégratif descendant », actuellement encouragé dans les textes officiels, plaident «pour une approche mixte» (2001: 106-109) et présentent des «outils orthographiques» nombreux et variés. Nous espérons que les démarches que nous avons présentées et mises en œuvre contribueront à clarifier les rapports entre les différentes approches de la langue dans les démarches d'apprentissage.

\section{Bibliographie}

BRISSAUD, C., BESSONNAT, D. (2001): L'orthographe au collège. Pour une autre approche, CRDP de Grenoble/Delagrave.

3. Voir Le Français aujourd'hui, 141, 2003. 
Chevrot, J.-P. (Dir. 1999): «L'orthographe et ses scripteurs », Langue française, 24.

DuCARD, D., HonvaulT, R., JAFFRE, J.-P. (1995): L'orthographe en trois dimensions, Paris, Nathan.

Fayol, M., Thévenin, M.-G., Totereau, C., Largy, P. (1997): Apprendre et gérer la morphologie du nombre à l'écrit, Liaisons HESO, 29-30, 93-108.

JAFrRÉ, J.-P. (1998): Procédures métagraphiques et acquisition de l'écrit, in J. Dolz et J.-C. Meyer (eds), Activités métalangagières et enseignement du français. Actes des journées d'études en didactique du français (Cartigny, 28 fevr.-1 $1^{\text {er }}$ mars 1997), Bern, Peter Lang, 47-62.

JAFFRÉ, J.-P., BessonnAT, D. (1993): Accord ou pas d'accord? Les chaines morphologiques, Pratiques, 77, Ecriture et Langue, 25-42.

Le Français aujourd'hui, 141, 2003 : Enseigner la langue de l'école au lycée (Actes du congrès de Besançon, mai 2002).

Pellat, J.-C. (2003): Les domaines de la description grammaticale, Le Français aujourd'hui, 141, Enseigner la langue de l'école au lycée (Actes du congrès de Besançon, mai 2002), 36-43.

Pellat, J.-C., Teste, G. (2001): Orthographe et écriture: pratique des accords, Strasbourg, CRDP d'Alsace.

TESTE, G. (2004): L'acquisition des chaines morphographiques d'accord au cycle 3 des écoles: aperçu d'une recherche action, in C. Vargas (dir.), Langue et étude de la langue. Approches linguistiques et didactiques, publication de l'Université de Provence, Aix, 333-340. 